\title{
A Note on Accurate and Efficient Higher Order Galerkin Time Stepping Schemes for the Nonstationary Stokes Equations
}

\author{
S. Hussain ${ }^{*}$, F. Schieweck and S. Turek
}

Institut für Angewandte Mathematik, TU Dortmund, Vogelpothsweg 87, D-44227 Dortmund, Germany

\begin{abstract}
In this note, we extend our recent work for the heat equation in [1] and describe and compare by means of numerical experiments the continuous Galerkin-Petrov (cGP) and discontinuous Galerkin (dG) time discretization applied to the nonstationary Stokes equations in the two-dimensional case. For the space discretization, we use the well-known LBB-stable quadrilateral finite element which consists of conforming biquadratic elements for the velocity and discontinuous linear elements for the pressure. We discuss implementation aspects as well as methods for solving the resulting block systems using monolithic multigrid solvers based on Vanka-type smoothers. By means of numerical experiments we compare the different time discretizations with respect to accuracy and computational costs. We show that the convergence behavior of the multigrid method is almost independent of the mesh size in space and the time step size which means (at least for these examples) that we have created an efficient solution process.
\end{abstract}

2000 Mathematics Subject Classification (MSC): 65M12, 65M55, 65M60.

Keywords: Discontinuous Galerkin method, Continuous Galerkin-Petrov method, Stokes equations, Multigrid solver.

\section{INTRODUCTION}

To solve time dependent partial differential equations, different time stepping schemes have been developed and analyzed. In practice, it is quite common to discretize first in space and then in time, which is known as the method of lines. This approach creates a system of ordinary differential equations which can be solved by one of the "good " state of the art ODE methods where "good" means stable, accurate and efficient. However, the spatial mesh points stay fixed in time which often is a restriction, for example, in the case where moving meshes are needed to approximate the solution properly. Thus, the method may have difficulties in changing the spatial mesh over the time steps. On the other hand, the Rothe method, which first discretizes in time, allows fully adaptive integration of time dependent PDEs. Two classes of time discretization schemes of variational type based on Rothe's method are the continuous GalerkinPetrov method (cGP) and the discontinuous Galerkin method (dG). These methods are of higher order due to their theoretical construction. Moreover, the cGP-method is Astable [2] and the dG-method is L-stable [3] for each polynomial degree $k$ in time. In our recent paper [1], we described both methods in detail for the heat equation and proposed an efficient multigrid method for solving the according block systems in each time step. For example, we have demonstrated by means of numerical test problems that the cGP(2)-method is of third order accurate in each time point and even of fourth order in the endpoints $t_{n}$ of the time

*Address correspondence to this author at the Institut für Angewandte Mathematik, TU Dortmund, Vogelpothsweg 87, D-44227 Dortmund, Germany; Tel: +49-(0) 231-755-7216; Fax: +49-(0) 231-755-5933;

E-mail: shafqat.hussain@math.uni-dortmund.de intervals whereas the $\mathrm{dG}(1)$-method has the order two in each time point and order three in the discrete time points $t_{n}$.

Let us mention some earlier work on these methods in literature. The approach of the cGP-method has already been used by Aziz and Monk [4] (but not under this name) for the linear heat equation. They have proved optimal error estimates as well as superconvergence results at the discrete points $t_{n}$ of the time mesh. In [2], the cGP-method was presented as a "discontinuous Galerkin Petrov" method where the name was chosen due to the fact that the test space is discontinuous in time and different from the ansatz space. The method was analyzed for the linear case in an abstract Hilbert space and for the non-linear case in the Euclidean space. In [1] the method was renamed into its final name "continuous Galerkin Petrov" method since the ansatz space is continuous in time contrary to the well-known "discontinuous Galerkin" method [3]. The dG-method in time has already a long tradition in literature, see e.g. the classical book [3] with all relevant historical references. Therein, for an abstract model problem, which includes again the heat equation, optimal error estimates and superconvergence results at the discrete time points are proven. In [1] we have compared for the heat equation, the cGP( $k)$-method, where the time polynomial ansatz is of degree $k$, with the $\mathrm{dG}(k-1)$-method where both ansatzand test-space are time polynomial of degree $k-1$. Since both methods require to solve a linear $k \times k$-block system in each time step, they have comparable computational costs concerning computing time and memory requirements. However, concerning the discretization error in time, the accuracy of the cGP( $k)$-method is one order higher than that of the $\mathrm{dG}(k-1)$-method. On the other hand, the $\mathrm{dG}(k-1)$ method is L-stable, i.e., it has better damping properties with 
respect to high frequency error components than the (only) A-stable cGP $(k)$-method.

In this paper, we extend our work on the heat equation in [1] to the nonstationary Stokes equations. The new difficulty is now that we have to treat a saddle point problem arising from the mixed formulation with velocity and pressure. The problem here is the question how to handle the pressure since there is no "direct" evolution due to a missing time derivative for the pressure. For each of the time discretizations cGP(1), cGP(2) and $\mathrm{dG}(1)$, we describe in detail our approach to derive a linear block-system on each time interval for the block-unknowns consisting of velocity and pressure vectors. The cGP(1)-method is very close to the well-known Crank-Nicolson scheme. Both methods differ only in the choice of the unknown that is solved for on each time interval and in the way how the numerical integration of the right hand side is done. The cGP(1)-method is accurate of order 2 in the whole time interval as it is known for the Crank-Nicolson scheme. In the cGP(2)-method as well as in the dG(1)-method, we have two block-unknowns on each time interval which have to be computed by solving a linear $2 \times 2$ block system. The cGP(2)-method is accurate of order 3 in the whole time interval and superconvergent of order 4 in the discrete time points $t_{n}$. The $\mathrm{dG}(1)$-method is of order 2 in the whole time interval and superconvergent of order 3 in the discrete time points. From the theoretical point of view, we would expect these orders of accuracy for the velocity since the velocity is the solution of an evolution problem in the subspace of the divergence-free functions. However, we have observed the orders of accuracy on the whole time interval also for the pressure. The situation becomes different if we look at the superconvergence of the pressure at special time points. Since we obtain superconvergence results for the velocity at the discrete time points $t_{n}$, it is also desirable to get the same for the pressure at these points which is practically important, for instance, for the computation of the hydrodynamic forces in CFD problems such as drag and lift. Due to the fact that we do not have an initial pressure, we cannot obtain the pressure in cGP-methods at the endpoints $t_{n}$ of the time intervals by using the same extrapolation as for the velocity. In order to achieve the superconvergence for the pressure at these points, we make use of suitable Lagrangian interpolation polynomials in the cGP-method. Then, the same technique is also applied to the $\mathrm{dG}(1)$-method.

Another important aim of this paper is to construct and test a suitable multigrid method that can solve the resulting linear block-systems in an efficient way. Here we apply a geometrical monolithic block-multigrid solver. We concentrate only on the related algorithmic and numerical aspects and omit theoretical investigations. Multigrid methods can be regarded as the most efficient solvers for the solution of large sparse linear systems, in particular for those arising from the discretization of PDEs where the condition number of the system matrix deteriorates with increasing problem size. In contrast to other iterative solvers (like for instance BiCGStab or GMRes), multigrid solvers converge with a rate which is independent of the mesh size and require computational costs which are only linearly dependent on the number of unknowns. The efficiency and the robustness of these solvers essentially depend on the smoothing operator. We employ a Vanka-type smoother which can be considered as block Gauß-Seidel method, where successively for each element of the mesh a local system is solved containing all velocity and pressure unknowns for all local time points of the time interval that belong to this element. Our numerical experiments confirm that this multigrid method (see also [5, 6]) converges at a rate which is almost independent of the mesh size in space and the time step size.

\section{THE cGP- AND dG-METHODS FOR THE STOKES EQUATIONS}

We consider the nonstationary Stokes equations, i.e. we want to find a velocity $\mathbf{u}: \Omega \times[0, T] \rightarrow \mathbb{R}^{2}$ and a pressure $p: \Omega \times[0, T] \rightarrow \mathbb{R}$ such that

$$
\begin{array}{ccc}
\partial_{t} \mathbf{u}-v \Delta \mathbf{u}+\nabla p=f, & \operatorname{div} \mathbf{u}=0 & \text { in } \quad \Omega \times(0, T), \\
\mathbf{u}=0 \quad \text { on } \quad \partial \Omega \times[0, T], & \mathbf{u}(x, 0)=\mathbf{u}_{0}(x) \quad \text { in } \quad \Omega \text { for } t=0,
\end{array}
$$

where $v$ denotes the viscosity, $f: \Omega \times(0, T) \rightarrow \mathbb{R}^{2}$ is the body force and $\mathbf{u}_{0}: \Omega \rightarrow \mathbb{R}^{2}$ the initial velocity field at time $t=0$. For simplicity, we assume homogeneous Dirichlet conditions at the boundary $\partial \Omega$ of a polygonal domain $\Omega \subset \mathbb{R}^{2}$. To make this problem well-posed, one needs to impose an additional condition on $p$, i.e., $\int_{\Omega} p d \Omega=0$.

The proposed time discretization schemes are accurate of higher order and have been studied for the heat equation in [1]. In particular, the cGP(1) and cGP(2)-method are accurate of order 2 and 3, respectively, in the whole time interval. Moreover, the cGP(2)-method shows a superconvergent behavior of order 4 in the discrete time points. The $\mathrm{dG}(1)$-method is of order 2 in the whole time interval and superconvergent of order 3 in the discrete time points. The purpose of this paper is to perform numerical tests to illustrate that we can get for these time discretization schemes the same accuracy as for the heat equation also in the case of the Stokes equations. To this end, we only concentrate on the algorithmic and numerical aspects. A rigorous analysis regarding theoretical aspects will be subject of a forthcoming paper.

We start with the time discretization of problem (1) which is of variational type. In the following, let $I=[0, T]$ denote the time interval with some positive final time $T$. For a function $\mathbf{u}: \Omega \times I \rightarrow \mathbb{R}^{2}$ and a fixed $t \in I$, we will denote by $\mathbf{u}(t):=\mathbf{u}(\cdot, t)$ the associated velocity function at time $t$ which is an element of a suitable function space $\mathbf{V}$. In case of the Stokes equations, this space is the Sobolev space $\mathbf{V}=\left(H_{0}^{1}(\Omega)\right)^{2}$. Similarly, we denote by $p(t):=p(\cdot, t)$ the associated pressure function at time $t$ which is an element of the function space $Q=L_{0}^{2}(\Omega)$ where

$$
L_{0}^{2}(\Omega)=\left\{q \in L^{2}(\Omega): \int_{\Omega} q d x=0\right\} .
$$


In the time discretization, we decompose the time interval $I$ into $N$ subintervals $I_{n}:=\left[t_{n-1}, t_{n}\right]$, where $n=1, \ldots, N$ and $0=t_{0}<t_{1}<\ldots<t_{N-1}<t_{N}=T$. The symbol $\tau$ will denote the time discretization parameter and will also be used as the maximum time step size $\tau:=\max _{1 \leq n \leq N} \tau_{n}$ where $\tau_{n}:=t_{n}-t_{n-1}$.

Then, for the cGP(k)-method, we approximate the solution $\mathbf{u}: I \rightarrow \mathbf{V}$ by means of a function $\mathbf{u}_{\tau}: I \rightarrow \mathbf{V}$ which is piecewise polynomial of order $k$ with respect to time, i.e., we are looking for $\mathbf{u}_{\tau}$ in the discrete time space

$\mathbf{X}_{\tau}^{k}:=\left\{\mathbf{u} \in C(I, \mathbf{V}):\left.\mathbf{u}\right|_{I_{n}} \in \mathbb{P}_{k}\left(I_{n}, \mathbf{V}\right) \quad \forall n=1, \ldots, N\right\}$,

where

$\mathbb{P}_{k}\left(I_{n}, \mathbf{V}\right):=\left\{\mathbf{u}: I_{n} \rightarrow \mathbf{V}: \mathbf{u}(t)=\sum_{j=0}^{k} \mathbf{U}^{j} t^{j}, \forall t \in I_{n}, \mathbf{U}^{j} \in \mathbf{V}, \forall j\right\}$.

We introduce the time discrete test space

$\mathbf{Y}_{\tau}^{k-1}:=\left\{\mathbf{v} \in L^{2}(I, \mathbf{V}):\left.\mathbf{v}\right|_{I_{n}} \in \mathbb{P}_{k-1}\left(I_{n}, \mathbf{V}\right) \quad \forall n=1, \ldots, N\right\}$

consisting of piecewise polynomials of order $k-1$ which are globally discontinuous at the end points of the time intervals. Similarly, we will use for the time discrete pressure $p_{\tau}$ an analogous ansatz space $X_{\tau}^{k}$, where the vector valued space $\mathbf{V}$ is replaced by the scalar valued space $Q$, and an analogous discontinuous test space $Y_{\tau}^{k-1}$.

Now, in order to derive the time discretization, we multiply the momentum equation in (1) with some suitable $I_{n}$-supported test functions $\mathbf{v}_{\tau} \in \mathbf{Y}_{\tau}^{k-1}$, integrate over $\Omega \times I_{n}$, use Fubini's Theorem and partial space integration of the terms $\Delta \mathbf{u}$ and $\nabla p$ and apply the $k$-point Gaussian quadrature rule for the evaluation of the time integrals. To determine $\left.\mathbf{u}_{\tau}\right|_{I_{n}}$ and $\left.p_{\tau}\right|_{I_{n}}$ we represent them by the polynomial ansatz

$\mathbf{u}_{\tau}(t):=\sum_{j=0}^{k} \mathbf{U}_{n}^{j} \varphi_{n, j}(t), \quad p_{\tau}(t):=\sum_{j=0}^{k} P_{n}^{j} \varphi_{n, j}(t)$,

where the "coefficients" $\left(\mathbf{U}_{n}^{j}, P_{n}^{j}\right)$ are elements of the Hilbert space $\mathbf{V} \times Q$ and the real functions $\varphi_{n, j} \in \mathbb{P}_{k}\left(I_{n}\right)$ are the Lagrange basis functions with respect to $k+1$ suitable nodal points $t_{n, j} \in I_{n}$ satisfying the conditions

$\varphi_{n, j}\left(t_{n, i}\right)=\delta_{i, j}, \quad i, j=0, \ldots, k$

with the Kronecker symbol $\delta_{i, j}$. For an easy treatment of the initial condition, we set $t_{n, 0}=t_{n-1}$. Then, the initial condition is equivalent to the condition

$\mathbf{U}_{n}^{0}=\left.\mathbf{u}_{\tau}\right|_{I_{n-1}}\left(t_{n-1}\right)$ if $n \geq 2$ or $\mathbf{U}_{n}^{0}=\mathbf{u}_{0}$ if $n=1$.

The other points $t_{n, 1}, \ldots, t_{n, k}$ are chosen as the quadrature points of the $k$-point Gaussian formula on $I_{n}$. This formula is exact if the function to be integrated is a polynomial of degree less or equal to $2 k-1$. We define the basis functions $\varphi_{n, j} \in \mathbb{P}_{k}\left(I_{n}\right)$ of (4) via the affine reference transformation $T_{n}: \hat{I} \rightarrow I_{n}$ where $\hat{I}:=[-1,1]$ and

$t=T_{n}(\hat{t}):=\frac{t_{n-1}+t_{n}}{2}+\frac{\tau_{n}}{2} \hat{t} \in I_{n} \quad \forall \hat{t} \in \hat{I}, n=1, \ldots, N$.

Let $\hat{\varphi}_{j} \in \mathbb{P}_{k}(\hat{I}), \quad j=0, \ldots, k$, denote the basis functions satisfying the conditions

$\hat{\varphi}_{j}\left(\hat{t}_{i}\right)=\delta_{i, j}, \quad i, j=0, \ldots, k$,

where $\hat{t}_{0}=-1$ and $\hat{t}_{i}, i=1, \ldots, k$, are the standard Gaussian quadrature points for the reference interval $\hat{I}$. Then, we define the basis functions on the original time interval $I_{n}$ by

$\varphi_{n, j}(t):=\hat{\varphi}_{j}(\hat{t})$ with $\hat{t}:=T_{n}^{-1}(t)=\frac{2}{\tau_{n}}\left(t-\frac{t_{n}-t_{n-1}}{2}\right) \in \hat{I}$.

At the end, we obtain the following time discrete $I_{n}$ problem of the cGP(k)-method $[1,2]$ :

Find on interval $I_{n}=\left[t_{n-1}, t_{n}\right)$ the $k$ unknown pairs of "coefficients" $\left(\mathbf{U}_{n}^{j}, P_{n}^{j}\right) \in \mathbf{V} \times Q, j=1, \ldots, k$, such that for all $i=1, \ldots, k$, it holds

$$
\left.\begin{array}{rlrl}
\sum_{j=0}^{k} \alpha_{i, j}\left(\mathbf{U}_{n}^{j}, \mathbf{v}\right)_{\Omega}+\frac{\tau_{n}}{2} a\left(\mathbf{U}_{n}^{i}, \mathbf{v}\right)+\frac{\tau_{n}}{2} b\left(\mathbf{v}, P_{n}^{i}\right) & =\frac{\tau_{n}}{2}\left(f\left(t_{n, j}\right), \mathbf{v}\right)_{\Omega} & \forall \mathbf{v} \in \mathbf{V}, \\
b\left(\mathbf{U}_{n}^{i}, q\right) & =0 \quad \forall q \in Q, &
\end{array}\right\}
$$

where $\tau_{n}$ denotes the length of the time interval $I_{n}$, $\mathbf{U}_{n}^{0}:=\mathbf{u}_{\tau}\left(t_{n-1}\right)$ for $n>1, \mathbf{U}_{1}^{0}:=\mathbf{u}_{0}$ and $(\cdot, \cdot)_{\Omega}$ the usual inner product in $L^{2}(\Omega)$. The bilinear forms $a(\cdot, \cdot)$ and $b(\cdot, \cdot)$ on $\mathbf{V} \times \mathbf{V}$ and $\mathbf{V} \times Q$, respectively, are defined as

$$
\begin{array}{ll}
a(\mathbf{u}, \mathbf{v}):=\int_{\Omega} \nabla \mathbf{u} \cdot \nabla \mathbf{v} d x & \forall \mathbf{u}, \mathbf{v} \in \mathbf{V}, \\
b(\mathbf{v}, p):=-\int_{\Omega} \nabla \cdot \mathbf{v} p d x & \forall \mathbf{v} \in \mathbf{V}, \quad p \in Q .
\end{array}
$$

A typical property of this cGP(k)-variant is that the initial pressure $P_{n}^{0}$ of the ansatz (4) does not occur in this formulation. This will be the reason for some problems to achieve superconvergence for the pressure approximation at the discrete time levels $t_{n}$.

In the following subsections, we specify the constants $\alpha_{i, j}$ of the cGP(k)-method for the cases $k=1$ and $k=2$ and we describe explicitly the well-known $\mathrm{dG}(1)$ approach.

\section{1. cGP(1)-Method}

We use the one-point Gaussian quadrature formula with the point $\hat{t}_{1}=0$ and $t_{n, 1}=t_{n-1}+\frac{\tau_{n}}{2}$. Then, we get $\alpha_{1,0}=-1$ and $\alpha_{1,1}=1$. Thus, equation (10) leads to the following equation for the "one" unknown $\mathbf{U}_{n}^{1}=\mathbf{u}_{\tau}\left(t_{n-1}+\frac{\tau_{n}}{2}\right) \in \mathbf{V}$ and $P_{n}^{1}=p_{\tau}\left(t_{n-1}+\frac{\tau_{n}}{2}\right) \in Q$ 


$$
\left.\begin{array}{rl}
\left(\mathbf{U}_{n}^{1}, \mathbf{v}\right)_{\Omega}+\frac{\tau_{n}}{2} a\left(\mathbf{U}_{n}^{1}, \mathbf{v}\right)+\frac{\tau_{n}}{2} b\left(\mathbf{v}, P_{n}^{1}\right) & =\frac{\tau_{n}}{2}\left(f\left(t_{n, 1}\right), \mathbf{v}\right)_{\Omega}+\left(\mathbf{U}_{n}^{0}, \mathbf{v}\right)_{\Omega} \quad \forall \mathbf{v} \in \mathbf{V} \\
b\left(\mathbf{U}_{n}^{1}, q\right) & =0 \quad \forall q \in Q .
\end{array}\right\}
$$

Once we have determined the solution $\mathbf{U}_{n}^{1}$ at the midpoint $t_{n, 1}$ of the time interval $I_{n}$, we get the solution at the next discrete time point $t_{n}$ simply by polynomial interpolation with the ansatz (4), i.e.,

$\mathbf{u}_{\tau}\left(t_{n}\right)=2 \mathbf{U}_{n}^{1}-\mathbf{U}_{n}^{0}$,

where $\mathbf{U}_{n}^{0}$ is the initial value at the time interval $\left[t_{n-1}, t_{n}\right)$ coming from the previous time interval $I_{n-1}$ or the initial value $\mathbf{u}_{0}$.

If we would replace $f\left(t_{n, 1}\right)$ by the mean value $\left(f\left(t_{n-1}\right)+f\left(t_{n}\right)\right) / 2$, which means that we replace the one-point Gaussian quadrature of the right hand side by the Trapezoidal rule, the resulting cGP(1)-method is equivalent to the wellknown Crank-Nicolson scheme.

\section{2. cGP(2)-Method}

Here, we use the 2-point Gaussian quadrature formula with the points $\hat{t}_{1}=-\frac{1}{\sqrt{3}}$ and $\hat{t}_{2}=\frac{1}{\sqrt{3}}$. Then, we obtain the coefficients

$$
\left(\alpha_{i, j}\right)=\left(\begin{array}{ccc}
-\sqrt{3} & \frac{3}{2} & \frac{2 \sqrt{3}-3}{2} \\
\sqrt{3} & \frac{-2 \sqrt{3}-3}{2} & \frac{3}{2}
\end{array}\right) \quad i=1,2, j=0,1,2 .
$$

On the time interval $I_{n}$, we have to solve for the two "unknowns"

$\left(\mathbf{U}_{n}^{j}, P_{n}^{j}\right)=\left(\mathbf{u}_{\tau}\left(t_{n, j}\right), p_{\tau}\left(t_{n, j}\right)\right) \in \mathbf{V} \times Q$ with $t_{n, j}:=T_{n}\left(\hat{t}_{j}\right)$ for $j=1,2$.

The corresponding coupled system reads:

$$
\left.\begin{array}{rl}
\alpha_{1,1}\left(\mathbf{U}_{n}^{1}, \mathbf{v}\right)_{\Omega}+\frac{\tau_{n}}{2} a\left(\mathbf{U}_{n}^{1}, \mathbf{v}\right)+\alpha_{1,2}\left(\mathbf{U}_{n}^{2}, \mathbf{v}\right)_{\Omega}+\frac{\tau_{n}}{2} b\left(\mathbf{v}, P_{n}^{1}\right) & =\ell_{1}(\mathbf{v}) \\
\alpha_{2,1}\left(\mathbf{U}_{n}^{1}, \mathbf{v}\right)_{\Omega}+\alpha_{2,2}\left(\mathbf{U}_{n}^{2}, \mathbf{v}\right)_{\Omega}+\frac{\tau_{n}}{2} a\left(\mathbf{U}_{n}^{2}, \mathbf{v}\right)+\frac{\tau_{n}}{2} b\left(\mathbf{v}, P_{n}^{2}\right) & =\ell_{2}(\mathbf{v}) \\
b\left(\mathbf{U}_{n}^{1}, q\right) & =0 \\
b\left(\mathbf{U}_{n}^{2}, q\right) & =0,
\end{array}\right\}
$$

which has to be satisfied for all $\mathbf{v} \in \mathbf{V}$ and $q \in Q$ with $\ell_{i}(\cdot)$ defined by

$$
\ell_{i}(\mathbf{v})=\frac{\tau_{n}}{2}\left(f\left(t_{n, i}\right), \mathbf{v}\right)_{\Omega}-\alpha_{i, 0}\left(\mathbf{U}_{n}^{0}, \mathbf{v}\right)_{\Omega} \quad i=1,2
$$

Once we have determined the solutions $\left(\mathbf{U}_{n}^{j}, P_{n}^{j}\right)$ at the Gaussian points in the interior of the interval $I_{n}$, we get the solution at the right boundary $t_{n}$ of $I_{n}$ again by means of polynomial interpolation from the ansatz (4), i.e.,

$\mathbf{u}_{\tau}\left(t_{n}\right)=\mathbf{U}_{n}^{0}+\sqrt{3}\left(\mathbf{U}_{n}^{2}-\mathbf{U}_{n}^{1}\right)$ where $\mathbf{U}_{n}^{0}$ is the initial value at the time interval $I_{n}$.

\section{3. dG(1)-Method}

In the dG(1)-method, velocity and pressure are approximated by a discontinuous piecewise linear ansatz space, i.e. $\left(\mathbf{u}_{\tau}, p_{\tau}\right) \in \mathbf{Y}_{\tau}^{1} \times Y_{\tau}^{1}$. On time interval $I_{n}$ we use the polynomial representation

$\mathbf{u}_{\tau}(t):=\sum_{j=1}^{2} \mathbf{U}_{n}^{j} \varphi_{n, j}(t), \quad p_{\tau}(t):=\sum_{j=1}^{2} P_{n}^{j} \varphi_{n, j}(t)$,

with the two "coefficients" $\left(\mathbf{U}_{n}^{j}, P_{n}^{j}\right) \in \mathbf{V} \times Q, j=1,2$, which are the values of $\mathbf{u}_{\tau}$ and $p_{\tau}$, respectively, at the points $t_{n, j} \in I_{n}$ of the 2 point Gaussian formula. The real functions $\varphi_{n, j} \in \mathbb{P}_{1}\left(I_{n}\right)$ are the linear Lagrange basis functions with respect to these two Gaussian points.

In order to present the method, we use the following constants for $i, j \in\{1,2\}$

$$
\left(\gamma_{i, j}\right)=\left(\begin{array}{cc}
1 & \frac{\sqrt{3}-1}{2} \\
\frac{-\sqrt{3}-1}{2} & 1
\end{array}\right), \quad\left(d_{i}\right)=\left(\begin{array}{c}
\frac{\sqrt{3}+1}{2} \\
\frac{-\sqrt{3}+1}{2}
\end{array}\right) .
$$

Then, on the time interval $I_{n}$, one has to determine the two "unknowns" $\left(\mathbf{U}_{n}^{j}, P_{n}^{j}\right) \in \mathbf{V} \times Q$ as the solution of the following coupled system:

$$
\left.\begin{array}{rl}
\gamma_{1,1}\left(\mathbf{U}_{n}^{1}, \mathbf{v}\right)_{\Omega}+\frac{\tau_{n}}{2} a\left(\mathbf{U}_{n}^{1}, \mathbf{v}\right)+\frac{\tau_{n}}{2} b\left(\mathbf{v}, P_{n}^{1}\right)+\gamma_{1,2}\left(\mathbf{U}_{n}^{2}, \mathbf{v}\right)_{\Omega} & =\ell_{1}(\mathbf{v}), \\
\gamma_{2,1}\left(\mathbf{U}_{n}^{1}, \mathbf{v}\right)_{\Omega}+\gamma_{2,2}\left(\mathbf{U}_{n}^{2}, \mathbf{v}\right)_{\Omega}+\frac{\tau_{n}}{2} a\left(\mathbf{U}_{n}^{2}, \mathbf{v}\right)+\frac{\tau_{n}}{2} b\left(\mathbf{v}, P_{n}^{2}\right) & =\ell_{2}(\mathbf{v}), \\
b\left(\mathbf{U}_{n}^{1}, q\right) & =0 \\
b\left(\mathbf{U}_{n}^{2}, q\right) & =0
\end{array}\right\}
$$

which has to be satisfied for all $\mathbf{v} \in \mathbf{V}$ and $q \in Q$ with $\ell_{i}(\cdot)$ defined by

$$
\ell_{i}(\mathbf{v})=\frac{\tau_{n}}{2}\left(f\left(t_{n, i}\right), \mathbf{v}\right)_{\Omega}+d_{i}\left(\mathbf{U}_{n}^{0}, \mathbf{v}\right)_{\Omega} \quad i=1,2
$$

Once we have solved the above system, we obtain $\mathbf{u}_{\tau}$ and $p_{\tau}$ at the time $t_{n}$ by means of the following linear interpolation

$\mathbf{u}_{\tau}\left(t_{n}\right)=\frac{\sqrt{3}+1}{2} \mathbf{U}_{n}^{2}-\frac{\sqrt{3}-1}{2} \mathbf{U}_{n}^{1}$ and $p_{\tau}\left(t_{n}\right)=\frac{\sqrt{3}+1}{2} P_{n}^{2}-\frac{\sqrt{3}-1}{2} P_{n}^{1}$.

\section{SPACE DISCRETIZATION BY FEM}

Next, in each time step, we apply a standard Galerkin finite element discretization with the so-called $Q_{2} / P_{1}^{\text {disc }}$ Stokes element, i.e., with biquadratic finite elements for the velocity and discontinuous piecewise linear elements for the pressure. This LBB-stable element pair leads to an $L_{2}$ approximation order of $O\left(h^{3}\right)$ for the velocity and $O\left(h^{2}\right)$ for the pressure where $h$ denotes the mesh size of the space grid. In the following, we will present the resulting block 
systems for the cGP(1)-, cGP(2)- and dG(1)-method which are used in our numerical experiments.

\section{1. cGP(1)-Method}

The corresponding $3 \times 3$ block system on each time interval $I_{n}$ reads: For given initial velocity coefficient vectors $\underline{\mathbf{U}}_{n}^{0}=\left(\underline{U}_{n}^{0}, \underline{V}_{n}^{0}\right)$, find $\underline{U}_{n}^{1}, \underline{V}_{n}^{1}$ and a scaled pressure vector $\underline{\tilde{P}}_{n}^{1}$ such that

$$
\begin{aligned}
\left(M+\frac{\tau_{n}}{2} A\right) \underline{U}_{n}^{1}+B_{1} \underline{\tilde{P}}_{n}^{1} & =\frac{\tau_{n}}{2} F_{n}^{1}+M \underline{U}_{n}^{0} \\
\left(M+\frac{\tau_{n}}{2} A\right) \underline{V}_{n}^{1}+B_{2} \underline{\tilde{P}}_{n}^{1} & =\frac{\tau_{n}}{2} F_{n}^{2}+M \underline{V}_{n}^{0} \\
B_{1}^{T} \underline{U}_{n}^{1}+B_{2}^{T} \underline{V}_{n}^{1} & =0
\end{aligned}
$$

where $\underline{\tilde{P}}_{n}^{1}:=\frac{\tau_{n}}{2} \underline{P}_{n}^{1}$, and $M, A$ and $B$ denote the mass, Laplacian and gradient matrices, respectively. Once we have determined the solution $\underline{U}_{n}^{1}, \underline{V}_{n}^{1}$ we compute the nodal vector $\underline{U}_{n+1}^{0}, \underline{V}_{n+1}^{0}$ of the discrete solution $u_{\tau, h}$ at the time $t_{n}$ by using the following linear extrapolation

$u_{\tau, h}\left(t_{n}\right) \sim \underline{U}_{n+1}^{0}=2 \underline{U}_{n}^{1}-\underline{U}_{n}^{0}, \quad v_{\tau, h}\left(t_{n}\right) \sim \underline{V}_{n+1}^{0}=2 \underline{V}_{n}^{1}-\underline{V}_{n}^{0}$.

\section{2. cGP(2)-Method}

The $6 \times 6$ block system on each time interval $I_{n}$ reads: For given initial velocity vectors $\underline{\mathbf{U}}_{n}^{0}=\left(\underline{U}_{n}^{0}, \underline{V}_{n}^{0}\right)$, find $\underline{U}_{n}^{1}, \underline{U}_{n}^{2}, \underline{V}_{n}^{1}, \underline{V}_{n}^{2}$ and scaled pressure vectors $\underline{\tilde{P}}_{n}^{1}, \tilde{\tilde{P}}_{n}^{2}$ such that $\left(\begin{array}{cccccc}3 M+\tau_{n} A & (2 \sqrt{3}-3) M & 0 & 0 & B_{1} & 0 \\ (-2 \sqrt{3}-3) M & 3 M+\tau_{n} A & 0 & 0 & 0 & B_{1} \\ 0 & 0 & 3 M+\tau_{n} A & (2 \sqrt{3}-3) M & B_{2} & 0 \\ 0 & 0 & (-2 \sqrt{3}-3) M & 3 M+\tau_{n} A & 0 & B_{2} \\ B_{1}^{T} & 0 & B_{2}^{T} & 0 & 0 & 0 \\ 0 & B_{1}^{T} & 0 & B_{2}^{T} & 0 & 0\end{array}\right)\left(\begin{array}{c}\underline{U}_{n}^{1} \\ \underline{U}_{n}^{2} \\ \underline{V}_{n}^{1} \\ \underline{V}_{n}^{2} \\ \tilde{P}_{n}^{1} \\ \tilde{P}_{n}^{2}\end{array}\right)=\left(\begin{array}{c}R_{n}^{1} \\ R_{n}^{2} \\ R_{n}^{3} \\ R_{n}^{4} \\ 0 \\ 0\end{array}\right)$

where $\underline{\tilde{P}}_{n}^{i}:=\tau_{n} \underline{P}_{n}^{i}$ and

$$
\begin{array}{ll}
R_{n}^{1}=\tau_{n} F_{n}^{1}+2 \sqrt{3} M \underline{U}_{n}^{0}, & R_{n}^{2}=\tau_{n} F_{n}^{2}-2 \sqrt{3} M \underline{U}_{n}^{0}, \\
R_{n}^{3}=\tau_{n} G_{n}^{1}+2 \sqrt{3} M \underline{V}_{n}^{0}, & R_{n}^{4}=\tau_{n} G_{n}^{2}-2 \sqrt{3} M \underline{V}_{n}^{0} .
\end{array}
$$

Here, we compute the nodal vector $\underline{U}_{n+1}^{0}$ and $\underline{V}_{n+1}^{0}$ of the fully discrete solution $u_{\tau, h}$ at the time $t_{n}$ by using the following quadratic extrapolation

$u_{\tau, h}\left(t_{n}\right) \sim \underline{U}_{n+1}^{0}=\underline{U}_{n}^{0}+\sqrt{3}\left(\underline{U}_{n}^{2}-\underline{U}_{n}^{1}\right)$,

$v_{\tau, h}\left(t_{n}\right) \sim \underline{V}_{n+1}^{0}=\underline{V}_{n}^{0}+\sqrt{3}\left(\underline{V}_{n}^{2}-\underline{V}_{n}^{1}\right)$.

\section{3. dG(1)-Method}

The analogous $6 \times 6$ block system on the time interval $I_{n}$ reads: For given initial velocity vector $\underline{\mathbf{U}}_{n}^{0}=\left(\underline{U}_{n}^{0}, \underline{V}_{n}^{0}\right)$, find $\underline{U}_{n}^{1}, \underline{U}_{n}^{2}, \underline{V}_{n}^{1}, \underline{V}_{n}^{2}$ and scaled pressure coefficient vectors $\underline{\tilde{P}}_{n}^{1}, \underline{\tilde{P}}_{n}^{2}$ such that

$$
\left(\begin{array}{cccccc}
2 M+\tau_{n} A & (\sqrt{3}-1) M & 0 & 0 & B_{1} & 0 \\
(-\sqrt{3}-1) M & 2 M+\tau_{n} A & 0 & 0 & 0 & B_{1} \\
0 & 0 & 2 M+\tau_{n} A & (\sqrt{3}-1) M & B_{2} & 0 \\
0 & 0 & (-\sqrt{3}-1) M & 2 M+\tau_{n} A & 0 & B_{2} \\
B_{1}^{T} & 0 & B_{2}^{T} & 0 & 0 & 0 \\
0 & B_{1}^{T} & 0 & B_{2}^{T} & 0 & 0
\end{array}\right)\left(\begin{array}{c}
\underline{U}_{n}^{1} \\
\underline{U}_{n}^{2} \\
\underline{V}_{n}^{1} \\
\underline{V}_{n}^{2} \\
\tilde{P}_{n}^{1} \\
\tilde{P}_{n}^{2}
\end{array}\right)=\left(\begin{array}{c}
R_{n}^{1} \\
R_{n}^{2} \\
R_{n}^{3} \\
R_{n}^{4} \\
0 \\
0
\end{array}\right)
$$

where $\underline{\tilde{P}}_{n}^{i}:=\tau_{n} \underline{P}_{n}^{i}$ and

$$
\begin{array}{ll}
R_{n}^{1}=\tau_{n} F_{n}^{1}+(\sqrt{3}+1) M \underline{U}_{n}^{0}, & R_{n}^{2}=\tau_{n} F_{n}^{2}+(-\sqrt{3}+1) M \underline{U}_{n}^{0}, \\
R_{n}^{3}=\tau_{n} G_{n}^{1}+(\sqrt{3}+1) M \underline{V}_{n}^{0}, & R_{n}^{4}=\tau_{n} G_{n}^{2}+(-\sqrt{3}+1) M \underline{V}_{n}^{0} .
\end{array}
$$

In this case, we compute the nodal vector $\underline{U}_{n+1}^{0}, \underline{V}_{n+1}^{0}$ and $\underline{P}_{n+1}^{0}$ of the left side limit of the fully discrete solution $u_{\tau, h}$ at the time $t_{n}$ by using the following linear extrapolation

$$
\begin{aligned}
& u_{\tau, h}^{-}\left(t_{n}\right) \sim \underline{U}_{n+1}^{0}=\frac{\sqrt{3}+1}{2} U_{n}^{2}-\frac{\sqrt{3}-1}{2} U_{n}^{1}, \\
& v_{\tau, h}^{-}\left(t_{n}\right) \sim \underline{V}_{n+1}^{0}=\frac{\sqrt{3}+1}{2} V_{n}^{2}-\frac{\sqrt{3}-1}{2} V_{n}^{1} .
\end{aligned}
$$

One can obtain the pressure at the discrete time points $t_{n}$ by using the same extrapolation

$$
p_{\tau, h}^{-}\left(t_{n}\right) \sim \underline{P}_{n+1}^{0}=\frac{\sqrt{3}+1}{2} P_{n}^{2}-\frac{\sqrt{3}-1}{2} P_{n}^{1} .
$$

\section{POSTPROCESSING FOR HIGH ORDER PRESSURE}

In many flow problems, often the hydrodynamic forces such as drag, lift etc, have to be calculated. These forces consist of functionals for velocity and pressure at the same discrete time points. Now, since we have superconvergence results for the velocity only at the discrete time points $t_{n}$, it is desirable to get a high order pressure at the same points. In contrast to the $\mathrm{dG}(1)$-method, we cannot obtain the pressure in cGP-methods at the discrete time points by using the same extrapolation as for velocity since this would involve the initial pressure which we do not have. In this section, we explain how to get higher order accuracy for the pressure in the cGP-methods at the discrete time points $t_{n}$ from the obtained pressure at the intermediate $k$ Gaussian points in the subinterval $\left[t_{n-1}, t_{n}\right]$. The same technique is then also applied for the $\mathrm{dG}(1)$-method which gives better results than the associated extrapolation. To do this, we use the Lagrangian interpolation polynomials to get the solution at time $t_{n}$ which we explain in the following for the cGP(1), cGP(2) and dG(1)-method, respectively.

\section{1. cGP(1)-Method}

We consider the left and right subinterval at time $t_{n}$. Let $t_{0}$ and $t_{1}$ be the intermediate Gaussian points in these subintervals where the solution is already known. We can construct the corresponding linear Lagrangian interpolation polynomial $L(t)$ such that $L\left(t_{i}\right)=p_{\tau}\left(t_{i}\right)$ for $i=0,1$. Once 
this linear polynomial $L(t)$ is obtained we can get the solution at the discrete time point $t_{n}$. In this way, one extra time step would be required to compute the solution at the end point $t=T$ of the simulation. The corresponding interpolation is visualized in Fig. (1).

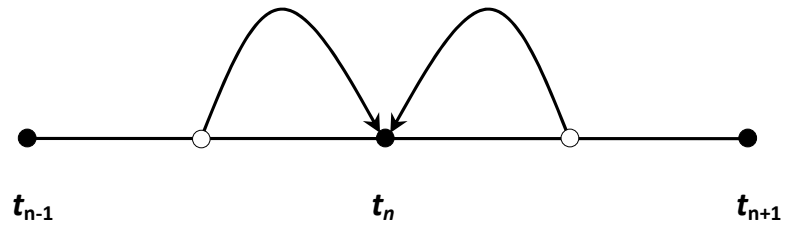

Fig. (1). Lagrange interpolation for pressure at the discrete time point $t_{n}$.

\section{2. cGP(2) and dG(1)-Method}

In case of $\mathrm{cGP}(2)$ or $\mathrm{dG}(1)$-method, we have two Gaussian points in each subinterval $I_{n}$. Let $t_{0}, t_{1}, t_{2}$ and $t_{3}$ be the four points in the neighboring subintervals at $t_{n}$. Now we construct the cubic Lagrangian polynomial passing through these four points. Once we have determined this polynomial $L(t)$ we obtain the solution at the next discrete time point $t_{n}$ (see Fig. 2). As in case of cGP(1), one more time step is needed to find the solution at the end point.

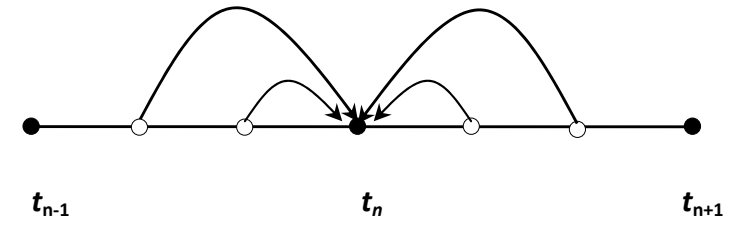

Fig. (2). Lagrange interpolation for pressure at the discrete time point $t_{n}$.

\section{SOLUTION OF THE LINEAR SYSTEMS}

The resulting linear systems in each time interval $\left[t_{n-1}, t_{n}\right]$, which are $6 \times 6$ block systems in the case of the $\mathrm{cGP}(2)$ and $\mathrm{dG}(1)$ approach and $3 \times 3$ block systems for the cGP(1)-method, are treated by using a geometrical multigrid solver with a local pressure Schur complement smoother (see $[6,7])$. Multigrid methods are known as the most efficient iterative methods for the solution of large linear systems arising from the discretization of partial differential equations, particularly of elliptic type. In this paper, we use the standard refinement scheme (see [6]) for the grid hierarchies, and for the smoothing operator, a cell centered Vanka like smoother is employed. Moreover, we use the canonical grid transfer routines regarding the chosen FEM space which treat both solution components separately in the case of the $\mathrm{cGP}(2)$ and $\mathrm{dG}(1)$ approaches (see [8] for the details, particularly regarding the grid transfer for the biquadratic finite elements). Finally, the coarse grid problem is solved by a direct solver.

\section{NUMERICAL RESULTS}

In this section, we perform several numerical tests in order to compare the accuracy of the proposed time discretization schemes. As a test problem we consider the
Stokes problem (1) with the domain $\Omega:=(0,1)^{2}$ and $v=1$. The prescribed velocity field $\mathbf{u}=\left(u_{1}, u_{2}\right)$ is

$$
\begin{aligned}
& u_{1}(x, y, t):=x^{2}(1-x)^{2}\left[2 y(1-y)^{2}-2 y^{2}(1-y)\right] \sin (10 \pi t), \\
& u_{2}(x, y, t):=-\left[2 x(1-x)^{2}-2 x^{2}(1-x)\right] y^{2}(1-y)^{2} \sin (10 \pi t),
\end{aligned}
$$

and the pressure distribution $p(x, y, t):=-\left(x^{3}+y^{3}-0.5\right)$ $(1.5+0.5 \sin (10 \pi t))$. The initial data is $\mathbf{u}_{0}(x, y)=\mathbf{u}(x, y, 0)$.

We apply the time discretization schemes cGP(1), $\mathrm{cGP}(2)$ and $\mathrm{dG}(1)$ with an equidistant time step size $\tau=T / N$. To measure the error (in time), the following discrete $L^{\infty}$-norm of a function $v: I \rightarrow L^{2}(\Omega)$ is used

$\|v\|_{\infty}:=\max _{1 \leq n \leq N}\left\|v^{-}\left(t_{n}\right)\right\|_{L^{2}(\Omega)}, \quad v^{-}\left(t_{n}\right):=\lim _{t \rightarrow t_{n}^{-0}} v(t), \quad t_{n}:=n \tau$.

The behavior of the standard $L^{2}$-norm $\|\cdot\|_{2}:=\|\cdot\|_{L^{2}\left(I, L^{2}(\Omega)\right)}$ and the discrete $L^{\infty}$-norm of the time discretization error $u(t)-u_{h, \tau}(t)$ for the velocity over the time interval $I=[0,1]$ can be seen in Tables $\mathbf{1}$ and $\mathbf{2}$, respectively. The estimated value of the experimental order of convergence (EOC) is also calculated and compared with the theoretical order of convergence for both velocity and pressure. All our numerical tests, where we compared the accuracy of our time discretization schemes are related to space mesh level 7 with equidistant $h=2^{-6}$.

We see that the cGP(2)-method is of order 3 in the $L^{2}$ norm and superconvergent of order 4 at the discrete time points $t_{n}$, while the $\mathrm{dG}(1)$-method is of order 2 in the $L^{2}$ norm and superconvergent of order 3 at the end points of the time intervals as expected from the theory. The cGP(1)method is of order 2 everywhere which is the same behavior as that of the well-known Crank-Nicolson scheme.

Now we show the accuracy of our time discretization schemes for the pressure. Here, we also illustrate the behavior of the $L^{2}$-norm $\|\cdot\|_{2}:=\|\cdot\|_{L^{2}\left(I, L^{2}(\Omega)\right)}$ and the discrete $L^{\infty}$-norm of the error in the pressure, respectively.

From Table 3, we observe that the experimental orders of convergence (EOC) coincide with the theoretical orders of convergence for corresponding time discretization schemes. Next, we want to analyze the behavior of $L^{\infty}$-error for the pressure. As we have already discussed, one can achieve the high order pressure at the discrete time points $t_{n}$ by using the Lagrangian interpolation polynomials symmetric at $t_{n}$. The behavior of the discrete $L^{\infty}$-norm of the error for pressure can be seen in Table 4.

We observe that the cGP(2)-method has superconvergent results of order 4 for pressure at the discrete time points $t_{n}$, while both the cGP(1) and dG(1)-method are of order 2 and 3 , respectively, at the end points of the time intervals as expected. 
Table 1. Error Norms $\left\|u-u_{h, \tau}\right\|_{2}$ for Velocity

\begin{tabular}{|c|c|c|c|c|c|c|}
\hline \multirow[b]{2}{*}{$1 / \tau$} & \multicolumn{2}{|c|}{ cGP(1) } & \multicolumn{2}{|c|}{ cGP(2) } & \multicolumn{2}{|c|}{ dG(1) } \\
\hline & $\left\|u-u_{h, \tau}\right\|_{2}$ & EOC & $\left\|u-u_{h, \tau}\right\|_{2}$ & EOC & $\left\|u-u_{h, \tau}\right\|_{2}$ & EOC \\
\hline 10 & $5.56 \mathrm{E}-03$ & & $4.40 \mathrm{E}-04$ & & $2.91 \mathrm{E}-03$ & \\
\hline 20 & $1.53 \mathrm{E}-03$ & 1.86 & $1.11 \mathrm{E}-04$ & 1.99 & $6.51 \mathrm{E}-04$ & 2.16 \\
\hline 40 & $3.93 \mathrm{E}-04$ & 1.97 & $1.33 \mathrm{E}-05$ & 3.06 & $1.82 \mathrm{E}-04$ & 1.84 \\
\hline 80 & $9.87 \mathrm{E}-05$ & 1.99 & $1.62 \mathrm{E}-06$ & 3.04 & 4.83E-05 & 1.91 \\
\hline 160 & $2.47 \mathrm{E}-05$ & 2.00 & $2.03 \mathrm{E}-07$ & 3.00 & $1.25 \mathrm{E}-05$ & 1.95 \\
\hline 320 & $6.18 \mathrm{E}-06$ & 2.00 & & & $3.17 \mathrm{E}-06$ & 1.98 \\
\hline 640 & $1.55 \mathrm{E}-06$ & 2.00 & & & $8.00 \mathrm{E}-07$ & 1.99 \\
\hline 1280 & $3.88 \mathrm{E}-07$ & 2.00 & & & $2.03 \mathrm{E}-07$ & 1.98 \\
\hline 2560 & $1.01 \mathrm{E}-07$ & 1.94 & & & & \\
\hline
\end{tabular}

Table 2. Error Norms $\left\|u-u_{h, \tau}\right\|_{\infty}$ for Velocity

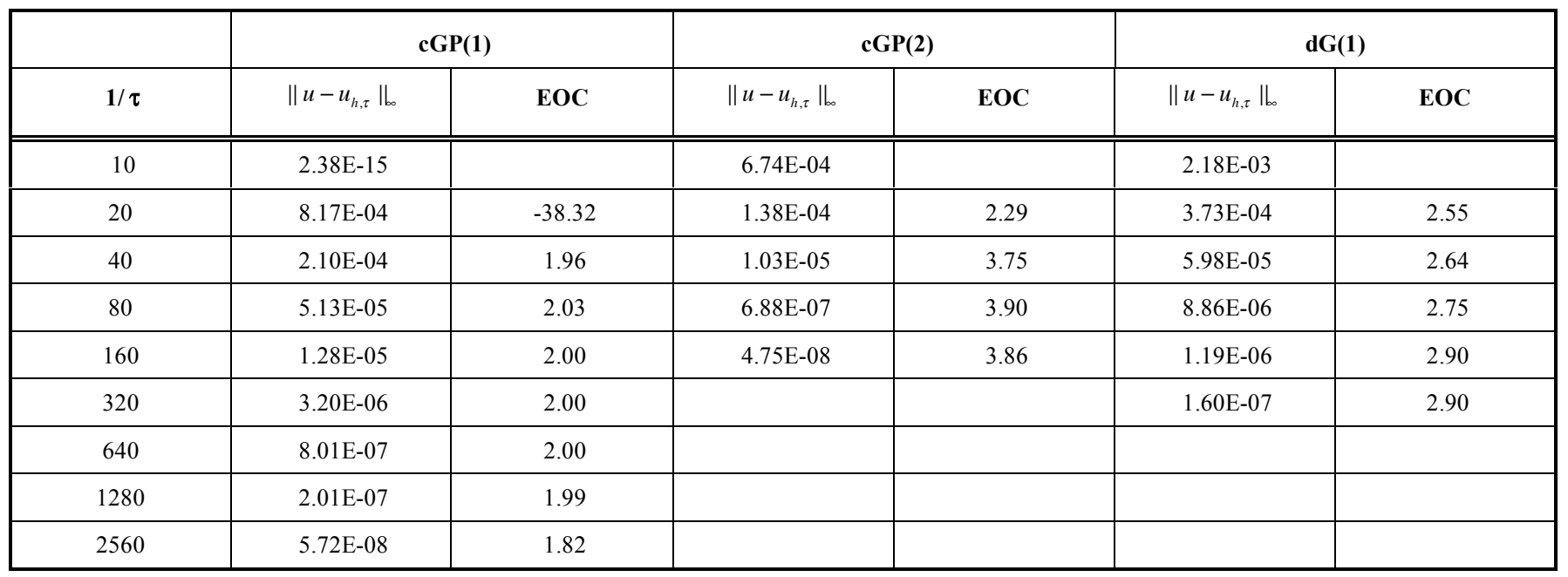

Table 3. Error Norms $\left\|p-p_{h, \tau}\right\|_{2}$ at the Gaussian Points for Pressure

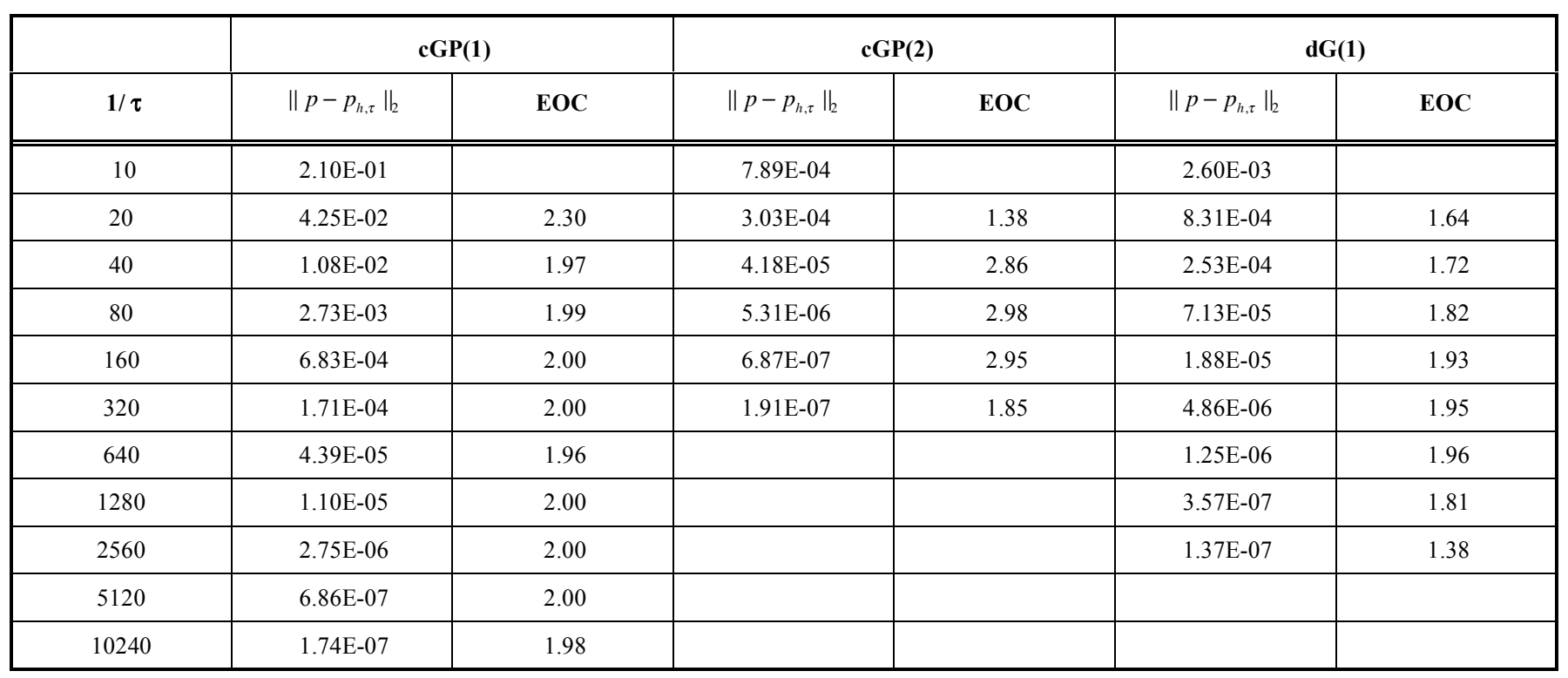


Since the error norms we compared so far contain both the spatial and time error, after a certain stage the space error becomes dominant. To see the accuracy for the time error more clearly, we now compute the norm $\left\|u_{h}-u_{h, \tau}\right\|_{b_{\infty}} \sim\left\|u_{h, \tau^{*}}-u_{h, \tau}\right\|_{\infty}$ by considering the reference time step size $\tau^{*}=1 / 2560$ for velocity and pressure for the cGP(2) and dG(1)-method.

One can see from Table 5 that the experimental orders of convergence for the $\mathrm{cGP}(2)$ and $\mathrm{dG}(1)$-methods are much more visible in the absence of spatial discretization errors.

Next, we perform numerical tests to analyze the corresponding behavior of the multigrid solver for the different time discretization schemes. As explained before, the solver uses a cell oriented Vanka type smoother and applies four pre- and post-smoothing steps. We present the averaged number of multigrid iterations per time step for solving the corresponding systems in Table 6. 'Lev' denotes the refinement level of the space mesh.
From Table 6, we see that the multigrid solver requires almost the same number of iterations for the different presented time discretization schemes. Moreover, the number of multigrid iterations remains fairly constant if we increase the refinement level of the space mesh. There is also no noticeable increase in the number of iterations if we decrease the time step (due to the non-diagonal mass matrix of $Q_{2}$ ). This means that the behavior of the multigrid solver is almost independent of the spatial mesh size and the time step.

Next, in order to measure and compare the efficiency of the multigrid solver for our time discretizations, we present in Table 7 the averaged CPU-time required for one solver iteration on a given space mesh level.

In these numerical tests, the multigrid solver has been implemented in our solver package FEAT2 (www.featflow.de). The CPU-times have been measured on an AMD Opteron 250 at $2.4 \mathrm{GHz}$. In Table 7, we observe that the CPU-time in case of $\operatorname{cGP}(2)$ or $\mathrm{dG}(1)$ is almost 3 times the CPU-time of cGP(1) for the multigrid solver. We also note that the CPU-time grows approximately by a factor of 4

Table 4. Error Norms $\left\|p-p_{h, \tau}\right\|_{\infty}$ for the Pressure Using Lagrange Interpolation

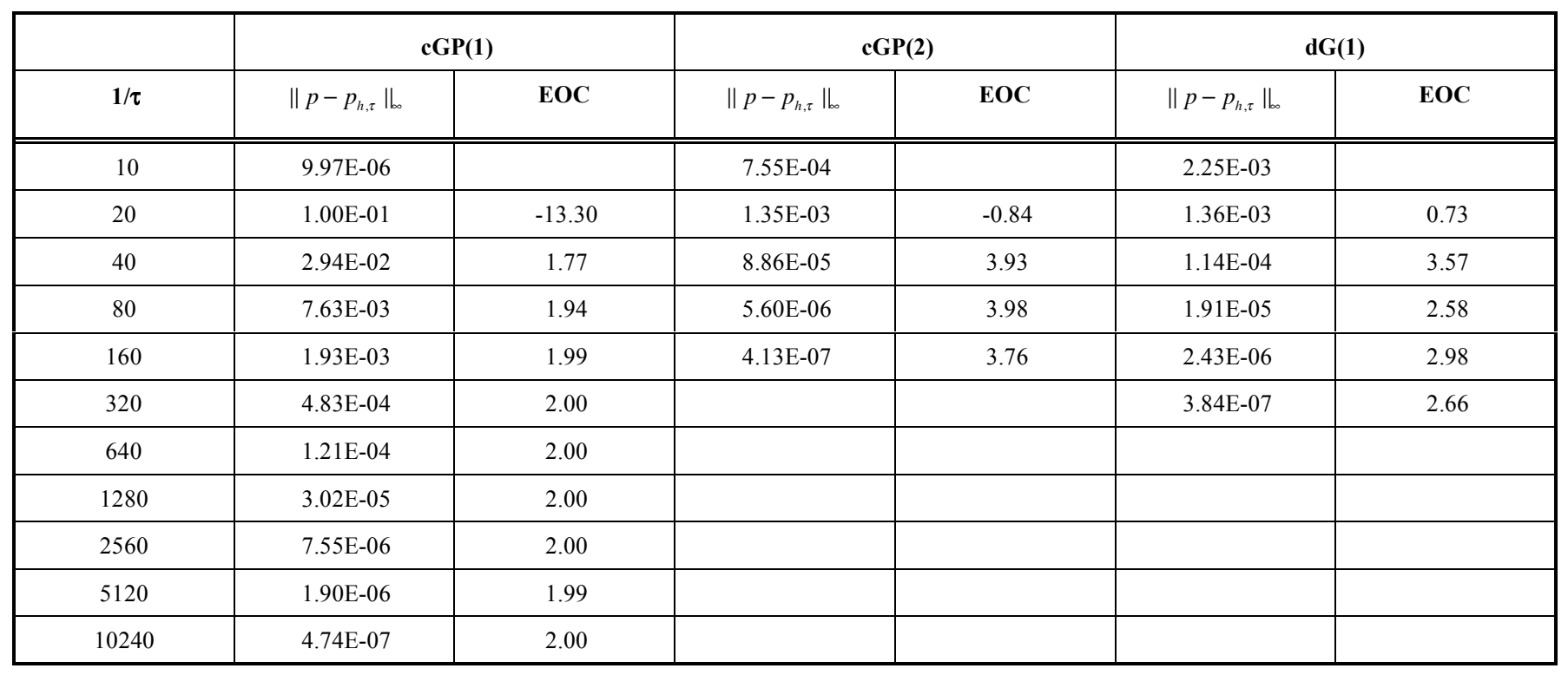

Table 5. Temporal Errors for Velocity and Pressure

\begin{tabular}{|c|c|c|c|c|c|c|c|c|}
\hline $1 / \tau$ & $\left\|u_{h}-u_{h, \tau}\right\|_{\infty}$ & EOC & $\left\|p_{h}-p_{h, \tau}\right\|_{\infty}$ & EOC & $\left\|u_{h}-u_{h, \tau}\right\|_{\infty}$ & EOC & $\left\|p_{h}-p_{h, \tau}\right\|_{\infty}$ & EOC \\
\hline 20 & $1.38 \mathrm{E}-04$ & 2.29 & $1.35 \mathrm{E}-03$ & -0.84 & $3.73 \mathrm{E}-04$ & 2.55 & $1.36 \mathrm{E}-03$ & 0.73 \\
\hline 40 & $1.03 \mathrm{E}-05$ & 3.75 & $8.86 \mathrm{E}-05$ & 3.93 & $5.98 \mathrm{E}-05$ & 2.64 & $1.14 \mathrm{E}-04$ & 3.57 \\
\hline 160 & 4.39E-08 & 3.97 & $3.51 \mathrm{E}-07$ & 4.00 & $1.19 \mathrm{E}-06$ & 2.90 & $2.42 \mathrm{E}-06$ & 2.63 \\
\hline 320 & $2.75 \mathrm{E}-09$ & 4.00 & 2.19E-08 & 4.00 & $1.55 \mathrm{E}-07$ & 2.94 & $3.46 \mathrm{E}-07$ & 2.80 \\
\hline 640 & $1.71 \mathrm{E}-10$ & 4.01 & $1.37 \mathrm{E}-09$ & 4.01 & $1.96 \mathrm{E}-08$ & 2.98 & $4.57 \mathrm{E}-08$ & 2.92 \\
\hline
\end{tabular}


as expected if we increase the space mesh level. These factors are nearly optimal since the number of space unknowns is increased by a factor of 4 if the level is increased by one.

Table 6. Averaged Multigrid Iterations Per Time Step for cGP(1) - cGP(2) - dG(1)

\begin{tabular}{|c|c|c|c|c|}
\hline Lev & $\boldsymbol{\tau}=\mathbf{1 / 2 0}$ & $\boldsymbol{\tau}=\mathbf{1 / 8 0}$ & $\boldsymbol{\tau}=\mathbf{1 / 3 2 0}$ & $\boldsymbol{\tau}=\mathbf{1 / 2 8 0}$ \\
\hline \hline 3 & $6-7-7$ & $8-9-8$ & $9-10-10$ & $10-11-10$ \\
\hline 4 & $9-8-9$ & $8-8-8$ & $8-10-9$ & $10-11-7$ \\
\hline 5 & $9-9-9$ & $8-8-8$ & $8-9-8$ & $9-10-9$ \\
\hline 6 & $10-10-9$ & $10-10-8$ & $8-8-8$ & $7-8-8$ \\
\hline 7 & $10-10-9$ & $10-10-10$ & $9-9-10$ & $8-8-8$ \\
\hline
\end{tabular}

Finally, we compare the time discretization schemes with respect to accuracy and numerical costs. Here, the multigrid solver uses four Vanka iterations in the pre- and postsmoothing step. The space discretization was done on mesh level 7. Table $\mathbf{8}$ shows, for different sizes of the time step $\tau$ and different time discretization schemes, the discrete $L^{\infty}$-norm and the total CPU-time for the computation in all time intervals. Due to its superconvergence of order 3 in the discrete time points, the $\mathrm{dG}(1)$-method is faster than cGP(1) which is only of order 2 .

One can see that, in order to achieve the accuracy of $10^{-7}$, we need the very small time step $\tau=1 / 2560$ for the cGP(1) while this accuracy can be already achieved with $\tau=1 / 160$ and $\tau=1 / 320$ in $\operatorname{cGP}(2)$ and $\mathrm{dG}(1)$-schemes, respectively. To compare the numerical costs per time step let us note that the number of multigrid iterations to solve one linear block system is approximately the same (about 8) for the three time discretization schemes. However, the costs of one multigrid iteration in the cGP(2) or $\mathrm{dG}(1)$ method is almost 3 times higher than in cGP(1). Nevertheless, for a desired accuracy of $10^{-7}$, the $\operatorname{cGP}(2)$ scheme is about 5 times faster than cGP(1) due to the much larger time step size required for $\mathrm{cGP}(2)$.

Next, we also compare our presented time discretization schemes with respect to accuracy and numerical costs for the pressure. To this end, we will only compare the accuracy

Table 7. CPU-Time Per Solver Iteration for Space Mesh Level=5,6,7, Respectively

\begin{tabular}{|c|c|c|c|c|c|c|c|c|c|}
\hline $1 / \tau$ & cGP(1) & cGP(2) & dG(1) & cGP(1) & cGP(2) & dG(1) & cGP(1) & cGP(2) & dG(1) \\
\hline 20 & 0.10 & 0.33 & 0.35 & 0.43 & 1.41 & 1.40 & 1.83 & 5.88 & 5.84 \\
\hline 40 & 0.11 & 0.33 & 0.33 & 0.47 & 1.40 & 1.43 & 2.03 & 6.02 & 6.14 \\
\hline 160 & 0.10 & 0.33 & 0.36 & 0.43 & 1.44 & 1.41 & 1.95 & 6.19 & 6.11 \\
\hline 320 & 0.10 & 0.33 & 0.36 & 0.53 & 1.45 & 1.55 & 1.94 & 6.23 & 5.88 \\
\hline 640 & 0.11 & 0.34 & 0.34 & 0.53 & 1.40 & 1.40 & 2.11 & 6.22 & 6.09 \\
\hline 1280 & 0.10 & 0.36 & 0.36 & 0.46 & 1.48 & 1.40 & 1.91 & 6.23 & 6.27 \\
\hline
\end{tabular}

Table 8. Error Norms $\left\|u-u_{h, \tau}\right\|_{\infty}$ and Total CPU-time to Achieve the Accuracy of $10^{-7}$ for Velocity Field

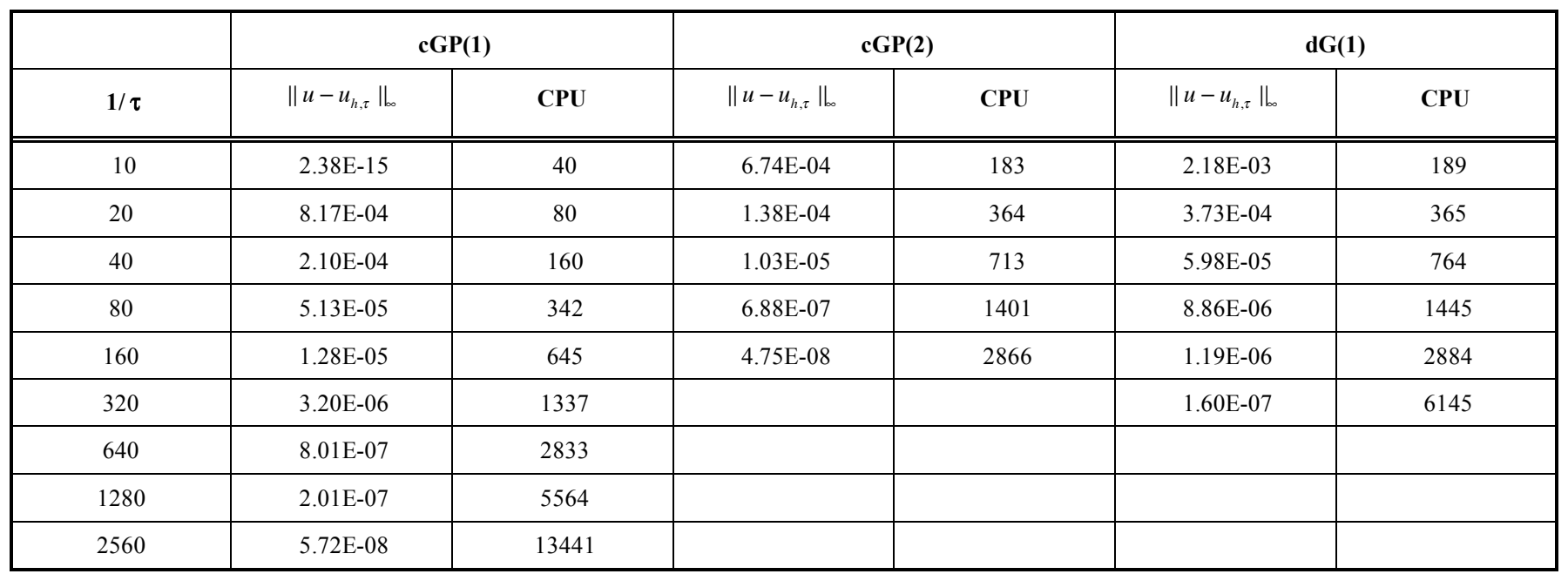


Table 9. Error Norms $\left\|p-p_{h, \tau}\right\|_{b_{\infty}}$ and Total CPU-Time to Achieve the Accuracy of $\mathbf{1 0}^{-6}$ for the Pressure Using Interpolation

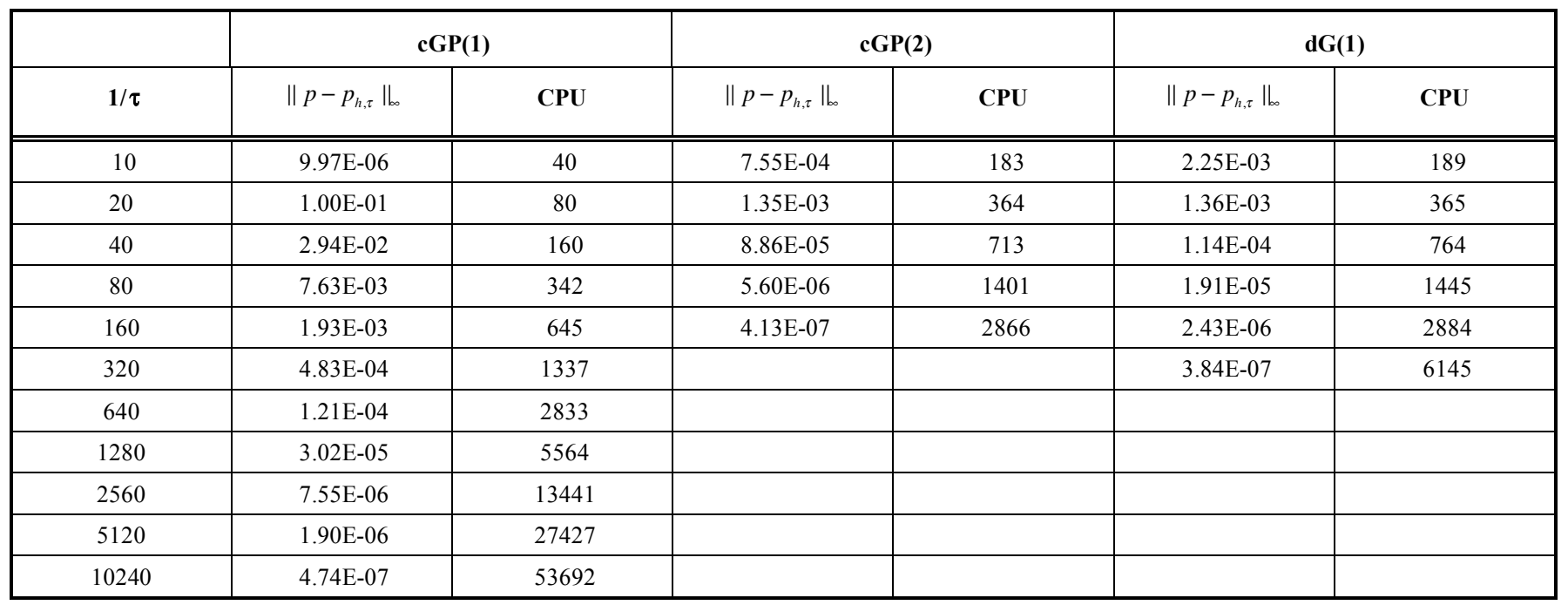

measured in the discrete $L^{\infty}$-norm. Here, the pressure is obtained at the discrete time points $t_{n}$ by using the Lagrangian interpolation procedure.

From Table 9, it can be seen that to achieve the accuracy of $10^{-6}$, the $\operatorname{cGP}(1)$ and $\mathrm{dG}(1)$-methods need very small time step sizes, i.e., $\tau=1 / 10240$ and $\tau=1 / 320$, while this accuracy has been already achieved with $\tau=1 / 160$ for the cGP(2) scheme. Hence, the cGP(2)-method always gives the accurate results for velocity and pressure in a much more efficient way.

At the end, to show that the proposed time discretization schemes can also efficiently handle the case when the solution approaches a steady state, we provide numerical tests with very large time steps. We consider problem (1) for $\Omega=(0,1)^{2}$ and the prescribed (time-independent) velocity

$$
\begin{aligned}
& u_{1}(x, y, t):=x^{2}(1-x)^{2}\left[2 y(1-y)^{2}-2 y^{2}(1-y)\right] \\
& u_{2}(x, y, t):=-\left[2 x(1-x)^{2}-2 x^{2}(1-x)\right] y^{2}(1-y)^{2}
\end{aligned}
$$

and the pressure distribution $p(x, y, t):=-\left(x^{3}+y^{3}-0.5\right)$. For these analytical solutions for $\mathbf{u}$ and $p$, we compute the corresponding right hand sides. As initial data we take $\mathbf{u}_{0}=0$.

Table $\mathbf{1 0}$ indicates for the multigrid method the number of solver iterations required for one time step which shows that there is no big difference in the number of solver iterations for time step size $\tau=10^{-6}$ up to $\tau=10^{6}$. This means that the behavior of the multigrid convergence is pretty robust with respect to very small as well as very large time steps.

\section{CONCLUSION}

We have described in detail the application of the continuous Galerkin-Petrov and discontinuous Galerkin time discretization schemes to the nonstationary Stokes equations. The spatial discretization is carried out by using biquadratic finite elements for velocity and discontinuous linear pressure. The presented linear block-systems for cGP(1), cGP(2) and $\mathrm{dG}(1)$ have been solved using a coupled geometrical multigrid method based on a Vanka-type blocksmoother. In our experiments, this multigrid-solver turned out to be of optimal computational complexity. From the numerical studies, we observed that the estimated experimental orders of convergence confirm the expected theoretical orders. Furthermore, the tests show that the cGP(2)-scheme provides significantly more accurate numerical solutions for both velocity and pressure than the other presented schemes cGP(1) and $\mathrm{dG}(1)$ which means that quite large time step sizes are allowed to gain highly accurate results. Secondly, all the presented time discretization schemes are also compared with respect to their numerical costs.

In our recent work, we currently extend these time discretization schemes to the Navier-Stokes equations to simulate complex time dependent flow problems in a very efficient way together with special Newton-multigrid techniques for the corresponding saddle point problems.

Table 10. Averaged Multigrid Iterations Per Time Step for cGP(1) - cGP(2) - dG(1)

\begin{tabular}{|c|c|c|c|c|c|}
\hline Lev & $\tau=\mathbf{1 0}^{-6}$ & $\tau=\mathbf{1 0}^{-3}$ & $\boldsymbol{\tau}=\mathbf{1}$ & $\boldsymbol{\tau}=\mathbf{1 0}^{\mathbf{3}}$ & $\boldsymbol{\tau}=\mathbf{1 0}^{\mathbf{6}}$ \\
\hline \hline 3 & $5-5-5$ & $5-5-5$ & $4-4-4$ & $4-4-4$ & $4-4-4$ \\
\hline 4 & $7-7-7$ & $5-6-6$ & $6-6-6$ & $6-6-6$ & $6-6-6$ \\
\hline 5 & $8-8-8$ & $6-7-7$ & $6-7-7$ & $7-7-7$ & $7-7-7$ \\
\hline 6 & $8-8-8$ & $6-7-7$ & $9-9-9$ & $9-9-9$ & $9-9-9$ \\
\hline 7 & $8-8-8$ & $7-7-7$ & $9-9-9$ & $9-9-9$ & $9-9-9$ \\
\hline 8 & $8-8-8$ & $8-8-8$ & $9-9-9$ & $9-9-9$ & $9-9-9$ \\
\hline
\end{tabular}




\section{ACKNOWLEDGEMENTS}

The authors want to express their gratitude to the German Research Association (DFG) and the Higher Education Commission (HEC) of Pakistan for their financial support of the study.

\section{CONFLICT OF INTEREST}

None declared.

\section{REFERENCES}

[1] Hussain S, Schieweck F, Turek S. Higher order Galerkin time discretizations and fast multigrid solvers for the heat equation. $\mathrm{J}$ Numer Math 2011; 19(1): 41-61.

[2] Schieweck F. A-stable discontinuous Galerkin-Petrov time discretization of higher order. J Numer Math 2010; 18(1): 25-57.
[3] Thomée V. Galerkin finite element methods for parabolic problems, volume 25 of Springer series in computational mathematics. 2nd ed. Berlin: Springer-Verlag 2006.

[4] Aziz AK, Monk P. Continuous infinite elements in space and time for the heat equation. Math Comp 1989; 52(186): 255-74.

[5] Hackbusch W. Multi-Grid methods and applications. Berlin: Springer-Verlag 1985

[6] Turek S. Efficient solvers for incompressible flow problems. An algorithmic and computational approach, volume 6 of Lecture Notes in Computational Science and Engineering. Berlin: Springer 1999.

[7] Wobker H, Turek S. Numerical studies of Vanka-type smoothers in Computational Solid Mechanics. AAMM 2009; 1(1): 29-55.

[8] Köster M, Turek S. The influence of higher order FEM discretisations on multigrid convergence. CMAM 2006; 6(2): 22132 .

(C) Hussain et al.; Licensee Bentham Open.

This is an open access article licensed under the terms of the Creative Commons Attribution Non-Commercial License (http://creativecommons.org/licenses/by-nc/ 3.0/) which permits unrestricted, non-commercial use, distribution and reproduction in any medium, provided the work is properly cited. 